\section{Prevalência, necessidade de tratamento e fatores predisponentes do traumatismo na dentição permanente de escolares de 11 a 13 anos de idade}

\author{
Prevalence, treatment needs, and predisposing \\ factors for traumatic injuries to permanent \\ dentition in 11-13-year-old schoolchildren
}

1 Programa de Pós-graduação
em Odontologia,
Universidade Federal
de Santa Catarina,
Florianópolis, Brasil.
2 Núcleo de Pesquisa
em Odontologia em Saúde
Coletiva, Universidade
do Sul de Santa Catarina,
Tubarão, Brasil.
3 Departamento de
Estomatologia, Universidade
Federal de Santa Catarina,
Florianópolis, Brasil.
4 Department of
Epidemiology and Public
Health, University College
London,London, U.K.
Correspondência
J. Traebert
Rua Coronel Teixeira
de Oliveria 380, Biguaçu,SC
88160-ooo, Brasil.
jtraebert@unisul.br

\section{Abstract}

The objective of this study was to assess the prevalence of traumatic injuries to the permanent incisors and the association with clinical predisposing factors and parents' schooling. A cross-sectional survey was conducted with schoolchildren aged 11 to 13 years in Biguaçu, Brazil. Dental examinations were conducted by a dentist, and the criteria for traumatic dental injuries used in the children's dental health survey in the United Kingdom were adopted. the study recorded the type of damage sustained, treatment performed or needed, the size of incisal overjet, and whether lip coverage was adequate. Socio-demographic data included sex, age, and parents' level of schooling. a total of 2,260 children were examined, and prevalence rates were $10.4 \%, 10.6 \%$, and $11.2 \%$ in 11,12 , and 13-year-old children, respectively. Treatment need was 6.3 interventions per thousand incisors. Male gender and overjet greater than $5 \mathrm{~mm}$ were significantly related to having a traumatic dental injury. Inadequate lip coverage and parents' educational level were not associated with dental trauma. The study concluded that male gender and incisal overjet greater than $5 \mathrm{~mm}$ are associated with the occurrence of dental injury.

Fractures; Tooth Injuries; Oral Health

\author{
J. Traebert 1,2 \\ I. C. S. Almeida 3 \\ C. Garghetti 3 \\ W. Marcenes 4
}

\section{Introdução}

Os altos índices de violência, acidentes de trânsito e uma maior participação das crianças em atividades esportivas têm contribuído para transformar o traumatismo dentário em um problema crescente em saúde pública 1 ao contrário da cárie dentária, cuja prevalência apresentou uma redução dramática nas últimas décadas 2. O traumatismo dentário deve ser considerado um problema importante não somente pelo fato de que sua prevalência é expressiva, principalmente em áreas de grande privação social e material 3, mas também devido ao seu alto impacto na qualidade de vida das crianças em termos de desconforto físico e psicológico, além do alto potencial de interferência negativa nas relações sociais 4 .

Poucos estudos de base populacional sobre prevalência de traumatismo na dentição permanente têm sido realizados na América Latina. A maioria dos dados publicados advém de estudos baseados em serviços de emergência, que não são representativos de uma determinada população. Sabe-se que estudos desta natureza provêem menos evidência epidemiológica do que estudos baseados em amostras aleatórias de uma população 5. Em uma revisão da literatura, baseada no MEDLINE, LILACS e BBO foram encontrados nove estudos, sendo três realizados na República Dominicana 6,7,8, um no México 9, e cinco no Brasil 10,11,12,13,14. Res- 
salta-se que os resultados dos estudos brasileiros são comparáveis, pois utilizaram metodologias semelhantes entre si e semelhantes à metodologia deste estudo.

$\mathrm{Na}$ determinação dos fatores etiológicos envolvidos no traumatismo dentário, é conhecido que uma medida de overjet incisal aumentada 15,16 e uma cobertura labial inadequada 15,17 são fatores predisponentes. Todavia, a influência de fatores sócio-econômicos não está suficientemente definida na literatura científica.

Assim, o objetivo deste estudo foi determinar a prevalência do traumatismo na dentição permanente em escolares de 11 a 13 anos de idade da cidade de Biguaçu, Estado de Santa Catarina, e observar possíveis associações com fatores clínicos predisponentes e nível de escolaridade dos pais ou responsáveis pelos escolares.

\section{Metodologia}

Foi realizado um estudo transversal incluindo escolares de 11 a 13 anos de idade de escolas públicas e privadas de Biguaçu, município da microrregião da Grande Florianópolis, com cerca de 40 mil habitantes 18 .

Todos os escolares da faixa etária deste estudo matriculados em 28 escolas do município foram convidados a participar do estudo. Autoridades locais como os secretários municipais de saúde e educação autorizaram a realização do estudo e forneceram nomes e endereços de cada escola do município e o número total de alunos matriculados, por idade.

O projeto desta pesquisa foi submetido ao Comitê de Ética em Pesquisa com Seres Humanos da Universidade Federal de Santa Catarina, obtendo autorização para sua realização pelo Parecer Consubstanciado 43/2001. Uma carta de consentimento livre e esclarecido foi enviada aos pais, conforme resolução específica do Conselho Nacional de Saúde sobre Ética em Pesquisa envolvendo seres humanos, explicando os objetivos, características, importância do estudo e solicitando autorização dos pais para participação de seu(sua) filho(a) no estudo. Quando os pais ou responsáveis aceitaram que seu(sua) filho(a) participasse do estudo, assinaram e devolveram o consentimento livre e esclarecido.

Os exames clínicos foram realizados por um cirurgião-dentista (J.T.), auxiliado por um anotador e um monitor. Previamente ao trabalho de campo, a equipe participou de um exercício de calibração envolvendo cinqüenta escolares da mesma faixa etária, de uma esco- la pública de uma cidade vizinha. A metodologia aplicada foi descrita em outra publicação 19 .

Todas as escolas foram visitadas duas vezes e as crianças examinadas na própria escola, no horário das aulas. Espelhos clínicos planos, sondas periodontais tipo CPI e gaze foram empacotados e esterilizados em quantidades suficientes para cada dia de trabalho. O examinador utilizou luvas descartáveis durante os exames, que incluíram os dentes incisivos superiores e inferiores e tecidos adjacentes. Foram anotados o tipo de dano traumático, o tratamento providenciado devido ao traumatismo, a necessidade de tratamento em razão do traumatismo e também o tamanho do overjet incisal e a adequabilidade da cobertura labial. Os critérios utilizados para registro do traumatismo dentário foram os mesmos utilizados no Children's Dental Health Survey do Reino Unido 16. Tais critérios incluíam fraturas, descoloração e perda do dente por causa do traumatismo na dentição permanente. A necessidade de tratamento devido ao traumatismo dentário foi anotado nos casos de presença de sinais de traumatismo não tratado ou perda da restauração realizada anteriormente por conta do traumatismo. Na ausência de outros sinais, pequenas fraturas de esmalte que não comprometessem a estética não foram incluídas na necessidade de tratamento. O tipo de tratamento necessário abrangeu restaurações adesivas, coroas unitárias, próteses móveis, tratamento endodôntico e clareamento. Se houvesse necessidade de outro tipo de tratamento, codificavase como "outro tipo de tratamento". O tipo de tratamento providenciado em virtude do traumatismo incluiu restauração adesiva, restauração adesiva e tratamento endodôntico, coroa unitária, prótese móvel. Se outro tipo de tratamento fosse encontrado, codificava-se como "outro tipo de tratamento". O overjet incisal foi codificado em menor ou igual a $5 \mathrm{~mm}$ ou maior que $5 \mathrm{~mm}$, após mensurar-se com a sonda periodontal tipo CPI a maior distância dos bordos incisais dos incisivos superiores até os bordos incisais dos correspondentes inferiores. Considerava-se como cobertura labial adequada quando os lábios se tocavam, cobrindo inteiramente os dentes anteriores, com o escolar lendo um documento mentalmente, sem saber que estava sendo observado. Outras informações coletadas incluíram idade, sexo e nível de escolaridade dos pais.

A variabilidade diagnóstica intra-examinador foi verificada por meio de exames duplos de $10 \%$ do total de escolares examinados. Para isto, foi utilizada a estatística Kappa tendo por 
base cada dente em cada uma das situações estudadas.

A análise dos dados foi realizada com o programa SPSS for Windows 10.0 e incluiu distribuição de freqüência e testes de associação. A significância estatística para a associação entre ocorrência de traumatismo dentário e idade, sexo, tamanho de overjet incisal, tipo de cobertura labial, nível de escolaridade do pai e da mãe foi determinada pela utilização do teste do qui-quadrado. As variáveis sexo, tamanho do overjet incisal, tipo de cobertura labial e nível de instrução do pai foram incluídas em um modelo de regressão logística múltipla com uso da técnica stepwise forward procedure. $\mathrm{O}$ nível de significância estabelecido foi $\mathrm{p}<0,05$.

Um estudo piloto foi realizado com uma amostra de trinta escolares com o objetivo de testar a metodologia do exame e aspectos administrativos. Os resultados demonstraram que a metodologia de pesquisa adotada era exeqüível na situação local.

\section{Resultados}

Um total de 2.260 crianças foram examinadas e entrevistadas em 28 diferentes escolas e a taxa de resposta foi de $90,6 \%$. As principais causas das não-respostas foram a não-autorização dos pais e a ausência das crianças em ambas as visitas às escolas. A concordância diagnóstica intra-examinador foi perfeita, uma vez que todos os valores de Kappa foram igual a 1,0. Isto aconteceu graças ao fato de os exames clínicos terem sido realizados por um examinador experiente e pelo fato dos critérios de diagnóstico do traumatismo dentário serem de mais fácil observação do que os de outras condições bucais.

Dos escolares examinados, 51,9\% eram do sexo masculino e $48,1 \%$ eram do sexo feminino. Em relação à idade, 36,0\% tinha 12 anos de idade, 32,0\% tinha 11 e 13 anos de idade (Tabela 1). A prevalência geral do traumatismo dentário foi de 10,7\%; as prevalências de acordo com as idades foram $10,4 \%, 10,6 \%$ e $11,2 \%$ aos 11,12 e 13 anos, respectivamente $(\mathrm{p}=0,864)$ (Tabela 1$)$.

Um total de 16,3 por mil incisivos apresentavam danos traumáticos. Fraturas de esmalte somente (7,7 por mil incisivos) e fraturas de esmalte e dentina (6,6 por mil incisivos), ambos sem sinais de envolvimento pulpar, foram os tipos de danos mais prevalentes. Outros tipos de danos clinicamente detectáveis, como fraturas com envolvimento pulpar, sinais de envolvimento pulpar sem fraturas ou dente perdido por causa do traumatismo foram menos prevalentes. Exclusivamente 15,6\% dos dentes com traumatismo apresentavam algum tipo de tratamento. O tipo de tratamento mais comumente encontrado foram as restaurações adesivas (1,4 incisivos por mil examinados). A necessidade de tratamento em virtude do traumatismo foi da ordem de 6,3 incisivos por mil examinados, e o tipo de tratamento mais necessário foram as restaurações adesivas $(5,7$ incisivos por mil examinados) (Tabela 2).

Os resultados da análise univariada confirmaram que meninos $(13,6 \%)$ tiveram mais traumatismo dentário do que as meninas $(7,6 \%)$ $(\mathrm{p}<0,001)$ (Tabela 1), e escolares com overjet incisal maior que $5 \mathrm{~mm}$ tiveram um índice maior de traumatismo dentário do que escolares com overjet até $5 \mathrm{~mm}(\mathrm{p}=0,003)$ (Tabela 1$)$. Além disso, houve uma tendência de escolares com cobertura labial inadequada terem uma maior prevalência de traumatismo dentário, mas a diferença não foi estatisticamente significativa ( $p=0,053$ ). Indicadores sociais como nível de educação do pai e da mãe não estiveram estatisticamente associados com a prevalência de traumatismo dentário $(\mathrm{p}=0,385$ e $\mathrm{p}=$ 0,485 respectivamente) (Tabela 1 ).

Os resultados da análise de regressão logística múltipla mostraram que somente o sexo do escolar ( $p<0,001)$ e o tamanho do overjet incisal ( $\mathrm{p}<0,001)$ mantiveram-se estatisticamente associados com a ocorrência do traumatismo dentário, após o ajuste pelas demais variáveis incluídas no modelo (Tabela 3).

\section{Discussão}

Este estudo transversal identificou uma prevalência de traumatismo dentário nos incisivos permanentes de $10,7 \%$ entre escolares de 11 a 13 anos de idade de Biguaçu. Esta prevalência é mais baixa que outros estudos brasileiros de base populacional realizados com amostras aleatórias e com os mesmos critérios de diagnóstico. Interessante é o fato de que esta prevalência é bastante inferior às relatadas em outras cidades catarinenses, onde a maioria dos estudos populacionais brasileiros de traumatismo dentário foram realizados. Cabe, contudo, relatar que Biguaçu, se comparada à Jaraguá do Sul, Blumenau e Florianópolis, onde outros estudos foram realizados, é a cidade que detém piores indicadores de desenvolvimento humano e privação social 18, e nenhum dos estudos realizados no Brasil mostrou maior prevalência relacionada a condições sócio-econômicas mais baixas. Como o delineamento deste estudo não foi apropriado para averiguar o motivo das diferenças nas prevalências entre 
Distribuição de freqüência do traumatismo nos incisivos permanentes em 2.260 escolares segundo idade, sexo, tamanho do overjet incisal, tipo de cobertura labial e nível de educação dos pais. Biguaçu, Santa Catarina, Brasil, 2001.

\begin{tabular}{|c|c|c|c|c|}
\hline & $\begin{array}{l}\text { Com traumatismo } \\
\text { dentário } n(\%)\end{array}$ & $\begin{array}{l}\text { Sem traumatismo } \\
\text { dentário n (\%) }\end{array}$ & $\begin{array}{l}\text { Total } \\
\text { n (\%) }\end{array}$ & $\begin{array}{l}\text { Valor de p para o } \\
\text { teste qui-quadrado }\end{array}$ \\
\hline \multicolumn{5}{|l|}{ Idade (anos) } \\
\hline 11 & $75(10,4)$ & $649(89,6)$ & $724(32,0)$ & 0,864 \\
\hline 12 & $86(10,6)$ & $727(89,4)$ & $813(36,0)$ & \\
\hline 13 & $81(11,2)$ & $642(88,8)$ & $723(32,0)$ & \\
\hline \multicolumn{5}{|l|}{ Sexo } \\
\hline Masculino & $159(13,6)$ & $1.014(86,4)$ & $1.173(51,9)$ & 0,001 \\
\hline Feminino & $83(7,6)$ & $1.004(92,4)$ & $1.087(48,1)$ & \\
\hline \multicolumn{5}{|c|}{ Tamanho do overjet incisal } \\
\hline$\leq 5 \mathrm{~mm}$ & $189(9,9)$ & $1.724(90,1)$ & $1.913(84,6)$ & 0,003 \\
\hline$>5 \mathrm{~mm}$ & $53(15,3)$ & $294(84,7)$ & $347(15,4)$ & \\
\hline \multicolumn{5}{|c|}{ Tipo de cobertura labial } \\
\hline Adequada & $167(10,0)$ & $1.509(90,0)$ & $1.676(74,2)$ & 0,053 \\
\hline Inadequada & $75(12,8)$ & $509(87,2)$ & $584(25,8)$ & \\
\hline \multicolumn{5}{|c|}{ Nível e educação do pai* } \\
\hline$\leq 8$ anos de estudo & $118(10,1)$ & $1.053(89,9)$ & $1.171(64,3)$ & 0,385 \\
\hline$>8$ anos de estudo & $62(9,5)$ & $589(90,5)$ & $651(35,7)$ & \\
\hline \multicolumn{5}{|c|}{ Nível de educação da mãe* } \\
\hline$\leq 8$ anos de estudo & $161(10,9)$ & $1.316(89,1)$ & $1.477(67,6)$ & 0,485 \\
\hline$>8$ anos de estudo & $76(10,7)$ & $632(89,3)$ & $708(32,4)$ & \\
\hline Total & $242(10,7)$ & $2.018(89,3)$ & $2.260(100,0)$ & \\
\hline
\end{tabular}

* Número de respostas menor em função do desconhecimento do nível de escolaridade dos pais.

as cidades, novas pesquisas devem ser realizadas com este objetivo.

As formas de traumatismo mais freqüentemente identificadas neste estudo foram as fraturas envolvendo apenas esmalte e fraturas envolvendo esmalte e dentina. Entretanto, mais importante a ser observado é que este estudo verificou também a necessidade de tratamento normativo além do dano traumático, o que é pouco comum. Os achados em relação à necessidade de tratamento confirmam outros estudos que incluíram esta variável 10,12 e que demonstraram uma sobrestimativa da necessidade de tratamento, quando estimada pelo número de lesões traumáticas não tratadas.

Em Biguaçu, como a maioria dos danos encontrados foi de pequena magnitude, 44,9\% das fraturas não tratadas não precisavam de nenhum tipo de tratamento. No entanto, os resultados mostraram que a necessidade de tratamento foi relativamente alta, podendo refletir negligência no tratamento do traumatismo dentário. Isto remete à reflexão dos motivos de tal negligência. Poder-se-ia sugerir o pouco acesso ao serviço odontológico por parte da maioria da população, como um importante determinante dos baixos índices de dentes traumatizados tratados. Todavia, cabe ressaltar que altas proporções de dentes traumatizados não tratados foram também relatados em países desenvolvidos, como o Reino Unido 3 onde o acesso ao serviço odontológico público e de boa qualidade é garantido a toda a população. Outro fator que poderia estar determinando os baixos índices de tratamento é o fato de o traumatismo dentário não ser uma doença; portanto, levando os pais a não atribuir a devida atenção ao evento. Porém, deve-se salientar a importância do tratamento dos dentes traumatizados em função de seu alto impacto na qualidade de vida dos indivíduos 4 e também pelo fato de que outras manifestações decorrentes do traumatismo podem acontecer a longo prazo. Outro aspecto que pode estar envolvido na 
negligência do tratamento é o baixo nível de conhecimento dos cirurgiões-dentistas no manejo do traumatismo dentário, tanto em países desenvolvidos 20, como também no Brasil 21. A grande variabilidade de metodologias aplicadas para a definição dos tipos de traumatismo, a falta de padronização de técnicas de tratamento e de protocolos-padrão de monitoramento das eventuais seqüelas futuras podem estar influenciando no preparo dos profissionais para lidar com o problema 22. A pouca importância historicamente alocada pela Odontologia ao traumatismo dentário pode ainda, segundo Andreasen \& Andreasen 15, ter acarretado conseqüências resultantes na negligência do tratamento por parte dos profissionais. Os autores destacam a característica de a terapia ser baseada empiricamente em casos já tratados, evidenciando a pouca pesquisa científica acerca do assunto; os traumatismos gerarem complicações cuja etiologia e tratamento serem pouco entendidos; o prognóstico a longo prazo ser pouco conhecido e, ainda, o fato de que o profissional que realiza o primeiro atendimento, muitas vezes não ser o profissional que realiza o monitoramento a longo prazo. Todas estas incertezas também podem estar interferindo no interesse do cirurgião-dentista em capacitar-se para o tratamento do traumatismo dentário.

Os resultados deste estudo mostraram que os escolares do sexo masculino sofreram significativamente mais traumatismo dentário do que escolares do sexo feminino $(\mathrm{p}<0,001)$. Este achado corrobora a maioria dos estudos publicados na literatura. Os meninos foram mais acometidos pelo traumatismo, provavelmente por serem mais ativos e realizarem atividades físicas mais fortes como esportes de contato físico sem a apropriada proteção, e brincadeiras rudes como lutas e outras, utilizando brinquedos e equipamentos com maior potencial de risco.

Um overjet maior que $5 \mathrm{~mm}$ aumenta a chance de sofrer traumatismo dentário, independentemente da influência das outras variáveis estudadas. Uma revisão sistemática utilizando metanálise afirmou que ter um tamanho de overjet maior do que $3 \mathrm{~mm}$ aumenta a chance de um indivíduo sofrer traumatismo dentário, independentemente de outras variáveis 23 . Estes resultados têm implicações importantes, especialmente para o serviço público, no sentido da necessidade de provisão de tratamento para as oclusopatias mais graves, dentre elas o overjet aumentado que, além de ter sido confirmado como importante fator predisponente ao traumatismo dentário, pode ter outros im-

\begin{tabular}{|c|c|c|}
\hline \multicolumn{3}{|c|}{$\begin{array}{l}\text { Proporção dos tipos de danos, tipos de tratamento providenciado } \\
\text { e necessidade de tratamento para o traumatismo por mil incisivos traumatizados } \\
\text { em } 2.260 \text { escolares de } 11 \text { a } 13 \text { anos de idade. Biguaçu, Santa Catarina, Brasil } \\
\text { ( } n=18.026 \text { incisivos). }\end{array}$} \\
\hline & $\begin{array}{l}\text { Freqüência } \\
\text { (n) }\end{array}$ & $\begin{array}{l}\text { Freqüência por } \\
\text { mil incisivos }\end{array}$ \\
\hline \multicolumn{3}{|l|}{ Traumatismo dentário não tratado } \\
\hline Fratura de esmalte & 140 & 7,7 \\
\hline Fratura de esmalte/dentina & 119 & 6,6 \\
\hline Fratura com envolvimento pulpar & 23 & 1,3 \\
\hline Envolvimento pulpar sem fratura & 9 & 0,5 \\
\hline Dente perdido & 2 & 0,1 \\
\hline Outros & 1 & 0,1 \\
\hline \multicolumn{3}{|l|}{ Tratamento providenciado } \\
\hline Restauração adesiva & 25 & 1,4 \\
\hline Restauração adesiva e tratamento endodôntico & 16 & 0,9 \\
\hline Coroa unitária & 1 & 0,1 \\
\hline Prótese móvel & 1 & 0,1 \\
\hline Outro tipo de tratamento & 1 & 0,1 \\
\hline \multicolumn{3}{|l|}{ Necessidade de tratamento } \\
\hline Restauração adesiva & 102 & 5,7 \\
\hline $\begin{array}{l}\text { Restauração adesiva, tratamento endodôntico } \\
\text { e clareamento }\end{array}$ & 8 & 0,4 \\
\hline Restauração adesiva e tratamento endodôntico & 3 & 0,1 \\
\hline Tratamento endodôntico e coroa unitária & 3 & 0,1 \\
\hline
\end{tabular}

pactos sobre a qualidade de vida das crianças e adolescentes.

Em relação à cobertura labial, a análise univariada deste estudo mostrou uma tendência de escolares com cobertura labial inadequada terem uma prevalência maior de traumatismo dentário $(\mathrm{p}=0,053)$, mas a diferença não foi estatisticamente significativa quando os resultados foram ajustados por outras variáveis estudadas $(p=0,50)$. Diversos estudos têm mostrado associação estatisticamente significativa entre o tipo de cobertura labial e prevalência de traumatismo dentário 1,11,24. Outros estudos, entretanto, não conseguiram demonstrar esta relação 4,10,12. Como a maioria dos estudos que incluíram esta variável são transversais, com metodologias diversas e apresentando resultados bastante divergentes, sugere-se que se estabeleça um protocolo de pesquisa padrão que possibilite estabelecer a real influência do tipo de cobertura labial na determinação do traumatismo dentário.

Neste estudo, nível de escolaridade do pai e da mãe não explicaram diferenças na prevalência do traumatismo dentário. No modelo de re- 
Regressão logística univariada e múltipla. Traumatismo dentário em escolares de 11 a 13 anos de idade. Biguaçu, Santa Catarina, Brasil, 2001.

\begin{tabular}{|c|c|c|c|c|}
\hline & OR bruta $($ IC95\%) & Valores de $p^{\star}$ & OR ajustada (IC95\%) & Valores de $p^{\star \star}$ \\
\hline \multicolumn{5}{|l|}{ Sexo } \\
\hline Feminino & 1,00 & & 1,00 & \\
\hline Masculino & $1,90(1,43-2,50)$ & 0,001 & $2,17(1,55-3,03)$ & 0,001 \\
\hline \multicolumn{5}{|c|}{ Tamanho do overjet incisal } \\
\hline$\leq 5 \mathrm{~mm}$ & 1,00 & & 1,00 & \\
\hline$>5 \mathrm{~mm}$ & $1,64(1,18-2,28)$ & 0,003 & $2,06(1,40-3,01)$ & 0,001 \\
\hline \multicolumn{5}{|c|}{ Tipo de cobertura labial } \\
\hline Adequada & 1,00 & & 1,00 & \\
\hline Inadequada & $1,33(1,00-1,78)$ & 0,053 & $1,13(0,79-1,61)$ & 0,497 \\
\hline \multicolumn{5}{|c|}{$\begin{array}{l}\text { Nível de escolaridade do pai } \\
\text { (anos de estudo) }\end{array}$} \\
\hline$>8$ & 1,00 & & 1,00 & \\
\hline$\leq 8$ & $0,94(0,68-1,30)$ & 0,939 & $1,05(0,75-1,46)$ & 0,783 \\
\hline
\end{tabular}

* valor de p bruto.

** valor de p para a variável ajustada pelas outras variáveis do modelo.

gressão logística múltipla, optou-se por incluir apenas uma variável de escolaridade dos pais, dado que o nível de escolaridade do pai e da mãe eram altamente correlacionados. Como foram testados separadamente modelos usando a escolaridade do pai e da mãe e os resultados foram semelhantes, somente o resultado do modelo incluindo escolaridade do pai foi apresentado para evitar colinearidade. O resultado encontrado neste estudo é semelhante ao encontrado em Jaraguá do Sul, Santa Catarina 10 e Cianorte, Paraná 13 , mas diferente daqueles de Blumenau, Santa Catarina 12, em que filhos de mães com mais de oito anos de escolaridade formal tiveram 1,6 vezes a chance de ter traumatismo dentário, se comparados com filhos de mães com menos de oito anos de escolaridade formal. Também em Belo Horizonte, Minas Gerais 11, crianças com melhores condições sócio-econômicas tiveram uma chance maior de sofrerem traumatismo dentário do que crianças com piores condições sócio-econômicas, o mesmo acontecendo com um estudo realizado na Jordânia 25 .

Ainda que este estudo não tenha mostrado associação, é interessante notar que nenhum outro estudo realizado no Brasil demonstrou uma maior prevalência em crianças de condições sócio-econômicas mais baixas. O maior risco de ocorrência de traumatismo dentário entre crianças de condição sócio-econômica mais alta, no Brasil e em outros países em desenvolvimento como a Jordânia, pode estar relacionado ao fato do maior acesso dessas crianças às piscinas, bicicletas, skates, patins, patinetes, prática de $s k i$ aquático, equitação e outros, do que crianças de condição sócio-econômica desfavorecida. Além disso, em países em desenvolvimento, mesmo crianças de famílias mais afluentes, praticam esportes e brincadeiras em ambientes pouco seguros, sem os necessários equipamentos de segurança. Estes resultados contraditórios sugerem que a interação entre a condição sócio-econômica individual e o ambiente físico pode exercer um papel importante na ocorrência do traumatismo dentário. Um maior acesso a bens e equipamentos de lazer está associado com crianças de maior nível sócio-econômico; todavia, se forem utilizados em ambientes inseguros, podem levar a uma maior ocorrência de traumatismo dentário. Assim, a direção da associação, se houver, pode depender do balanço entre estes fatores. Portanto, mais pesquisas devem ser realizadas com o objetivo de elucidar a relação entre status sócio-econômico e traumatismo dentário. 


\section{Resumo}

Este estudo objetivou determinar a prevalência do traumatismo dentário na dentição permanente e observar associações com fatores predisponentes. Foi realizado um estudo transversal com escolares de 11 a 13 anos de Biguaçu, Santa Catarina, Brasil. Os exames foram realizados por um cirurgião-dentista utilizando os mesmos critérios do Children's Dental Health Survey do Reino Unido. Foram observados tipo de dano, tratamento providenciado e necessidade, overjet incisal, adequabilidade da cobertura labial, idade, sexo e nível de educação dos pais. Foram examinadas 2.260 crianças e as prevalências encontradas foram: 10,4\%, 10,6\% e 11,2\% aos 11, 12 e 13 anos, respectivamente. A necessidade de tratamento foi de 6,3 incisivos por mil examinados. Escolares do sexo masculino e com overjet maior que $5 \mathrm{~mm}$ tiveram mais traumatismo dentário do que escolares do sexo feminino e com overjet incisal até $5 \mathrm{~mm}$. Cobertura labial inadequada e nível de educação dos pais não estiveram estatisticamente associados com o traumatismo dentário. Concluiu-se que ser do sexo masculino ou ter um overjet incisal maior do que $5 \mathrm{~mm}$ aumenta a chance de sofrer traumatismo dentário.

Fraturas; Traumatismos Dentários; Saúde Bucal

\section{Colaboradores}

J. Traebert, I. C. S. Almeida e W. Marcenes participaram da etapas de elaboração do projeto de pesquisa, da análise dos dados e da redação do artigo; J. Traebert e C. Garguetti realizaram a coleta de dados clínicos e não clínicos; C. Garguetti também participou das etapas de digitação dos dados, da análise dos dados e da redação do artigo.

\section{Referências}

1. Marcenes W, Beiruti N, Tayfour D, Issa S. Epidemiology of traumatic dental injuries to permanent incisors of school-children aged 9 to 12 in Damascus, Syria. Endod Dent Traumatol 1999; 15:117-23.

2. Andreasen JO, Andreasen FM. Textbook and color atlas of traumatic injuries to the teeth. 3rd Ed. Copenhagen: Munsksgaard; 1994.

3. Marcenes W, Murray S. Social deprivation and traumatic dental injuries among 14 year old schoolchildren in Newham, London. Endod Dent Traumatol 2000; 16:1-5.

4. Cortes MIS, Marcenes W, Sheiham A. Impact of traumatic injuries to the permanent teeth on the oral health-related quality of life of 12-14-yearold children. Community Dent Oral Epidemiol 2002; 30:193-8.

5. Hennekens $\mathrm{CH}$, Buring JE. Epidemiology in medicine. Boston: Little Bronw and Co.; 1987.

6. García-Godoy F, Sánchez R, Sánchez JR. Traumatic dental injuries in a sample of Dominican schoolchildren. Community Dent Oral Epidemiol 1981; 9:193-7.

7. García-Godoy F, Morban-Laucer F, Corominas LR, Franjul RA, Noyola M. Traumatic dental injuries in schoolchildren from Santo Domingo. Community Dent Oral Epidemiol 1985; 13:177-9.

8. García-Godoy F, Dipres FM, Lora IM, Vidal ED. Traumatic dental injuries in children from private and public schools. Community Dent Oral Epidemiol 1986; 14:287-90.

9. Sánchez AV, García-Godoy F. Traumatic dental injuries in 3 to 13 year old boys in Monterrey, Mexico. Endod Dent Traumatol 1990; 6:63-5.

10. Marcenes W, Alessi ON, Traebert J. Causes and prevalence of traumatic injuries to the permanent incisors of school-children aged 12 years in Jaragua do Sul, Brazil. Int Dent J 2000; 50:8792.

11. Cortes MIS, Marcenes W, Sheiham A. Prevalence and correlates of traumatic dental injuries to the teeth of schoolchildren aged 9 to 14 in Belo Horizonte, Brazil. Endod Dent Traumatol 2000; 17:226.

12. Marcenes W, Zabot NE, Traebert J. Socio-economic correlates of traumatic injuries to the permanent incisors in schoolchildren aged 12 years in Blumenau, Brazil. Endod Dent Traumatol 2001; 17:222-6.

13. Nicolau B, Marcenes W, Sheiham A. Prevalence, causes and correlates of traumatic dental injuries among 13-years-olds in Brazil. Endod Dent Traumatol 2001; 17:213-7.

14. Traebert J, Peres MA, Blank V, Boëll RS, Pietrusa JA. Prevalence of traumatic dental injury and associated factors among 12-year old school children in Florianópolis, Brazil. Dent Traumatol 2003; 19:15-8.

15. Andreasen JO, Andreasen FM. Dental trauma: quo vadis. Tandlaegebladet 1989; 93:381-4.

16. O'Brien M. Children's dental health in the United Kingdom 1993. In: Report of dental survey, Office of Population Censuses and Surveys. London: Her Majesty's Stationery Office; 1994. 
17. O'Mullane DM. Some factors predisposing to injuries to permanent incisors in school children. Br Dent J 1973; 134:328-32.

18. Fundação Instituto Brasileiro de Geografia e Estatística. Banco de dados. http://www.ibge.gov. br/home/estatistica/população/censo2000/uni verso.php?tipo $=31$ \&paginaatual $=1 \& u f=42 \&$ letra $=$ B $($ acessado em 02/Jul/2002).

19. Peres MA, Traebert J, Marcenes W. Calibração de examinadores para estudos epidemiológicos de cárie dentária. Cad Saúde Pública 2001; 17:153-9.

20. Hamilton FA, Hill FJ, Holloway PJ. An investigation of dento-alveolar trauma and its treatment in an adolescent population. Part 1: The prevalence and incidence of injuries and the extent and adequacy of treatment received. Br Dent J $1997 ; 182: 91-5$
21. Armênio R. Conhecimento dos cirurgiões-dentistas do meio-oeste catarinense sobre traumatismo dental [Monografia de Especialização]. Joaçaba: Escola de Aperfeiçoamento Profissional, Associação Brasileira de Odontologia; 2001.

22. Andreasen JO. Challenges in clinical dental trauma. Endod Dent Traumatol 1985; 1:454-5

23. Nguyen QV. A systematic review of the relationship between overjet size and traumatic dental injuries. Eur J Orthod 1999; 21:503-15.

24. Petti S, Cairella G, Tarsitani G. Childhood obesity: a risk factor for traumatic injuries to anterior teeth. Endod Dent Traumatol 1997; 13:285-8.

25. Jamani KD, Fayyad MA. Prevalence of traumatized permanent incisors in Jordanian children according to age, sex and socio-economic class. Odontostomatol Trop 1991; 14:17-20.

Recebido em 25/Abr/2002

Versão final reapresentada em 16/Jun/2003

Aprovado em 23/Out/2003 